\title{
Das Bundesamt für Landwirtschaft
}

\section{Ein Rückblick}

Das Bundesamt für Landwirtschaft (BLW) blickt auf eine nun mehr als 100 jährige Geschichte zurück. Davon zeugt die Jubiläumsschrift, welche im Jahre 1982 erschien. Das Amt besteht seit dem 1. Januar 1882, damals geschaffen als "Abtheilung Landwirthschaft» des "Handels- und Landwirthschaftsdepartements».

\section{Stellung und allgemeine Aufgabe des BLW}

Mit der Vorbereitung und der Durchführung der Agrarpolitik der Schweiz befassen sich im Bund zahlreiche Organe: Bundesrat, Eidgenössisches Volkswirtschaftsdepartement, zu dem das BLW gehört, weitere Departemente mit ihren Ämtern, Bundesversammlung, Gerichte, zahlreiche Organisationen. Dazu kommen die Kantone mit ihrer Legislative und Exekutive.

Das BLW ist dasjenige Amt der Bundesverwaltung, das sich ausschließlich und am umfassendsten mit der Agrarpolitik des Bundes befaßt. Seine Aufgabe ergibt sich einerseits aus der Agrarpolitik des Bundes überhaupt (siehe unten) und sodann aus den Aufgaben anderer Organe sowie Organisationen und der Kantone.

Das BLW hat im einzelnen verschiedene Aufgaben. Sie sind allgemein in der Verordnung vom 9. Mai 1979 des Bundesrates über die Aufgaben der Departemente, Gruppen und Ämter umschrieben (Art. 13 Zif. 4; SR 172.010.15), dazu aber auch in Gesetzen und Verordnungen.

\section{Die agrarpolitische Aufgabe des Bundes}

Die Aufgaben des Bundes nahmen im Laufe der Jahrzehnte im Bereich der Landwirtschaft - wie auch in den andern Gebieten des öffentlichen Lebens stets zu. Stand früher als Zweck dieses Wirtschaftszweiges fast ausschließlich die Versorgung mit Lebensmitteln in genügender Menge, in guter Qualität und zu angemessenen Preisen in Friedens- sowie in Krisen- und Kriegszeiten im Vordergrund - man denke an den Ersten und den Zweiten Weltkrieg -, so sind heute der Schutz von Landschaft und Umwelt sowie die Erhaltung der Besiedelung auch abgelegener Gebiete mehr in den Vordergrund getreten. Zur Agrarpolitik gehört sodann auch das staatspolitische Ziel der Erhaltung des Bauernstandes als eines Berusstandes neben anderen Berufsgruppen.

Die Rechtsgrundlagen der Agrarpolitik des Bundes finden sich in der Verfassung, in Gesetzen und Verordnungen sowie sodann in Staatsverträgen der Schweiz mit ausländischen Staaten oder Staatengruppen, wie den Europäischen Gemeinschaften, Staaten der Europäischen Freihandelsassoziation (EFTA). Die wichtigste Verfassungsbestimmung ist jene, wonach der Bund befugt ist, "zur Erhaltung eines gesunden Bauernstandes und einer leistungsfähigen Landwirtschaft sowie zur Festigung des bäuerlichen Grundbesitzes» die erforderlichen Vorschriften zu erlassen (BV Art. $31^{\text {bis }}$ Abs. 3 Bst. b). Das grundlegende Gesetz unter vielen - ist das Landwirtschaftsgesetz vom 3. Oktober 1951 (SR 910.1), etwa neben dem Bundesgesetz über Investitionskredite und Betriebshilfe in der Landwirtschaft, den Milchwirtschaftsbeschlüssen.

\section{Die Mittel der Agrarpolitik}

Die Instrumente zur Verwirklichung der Agrarpolitik sind sehr mannigfaltig. Dazu gehören zunächst alle jene Vorkehren der Grundlagenverbesserung, wie Forschung, Bildung, Beratung, Zucht und Züchtung, Verbesserung der Struktur, sodann etwa direkte $\mathrm{Maß}$ nahmen zur Erhaltung der landwirtschaftlichen Betriebe, wie etwa das bäuerliche Erbrecht und das Pachtrecht. Wesentlich und bemerkbar sind die zahlreichen Vorkehren zur Sicherung angemessener Preise und des Absatzes sowie zur Lenkung der Produktion (Beiträge zur Stützung der Produzentenpreise und zur Verbilligung der Erzeugnisse, Einfuhrbeschränkungen, Übernahmepflichten, Pflanzverbote, Stallbauverbote usw.). Sozialpolitische Maßnahmen, wie etwa Familienzulagen, ergänzen diesen Sektor. Polizeiliche Maßnahmen sollen die Landwirtschaft selbst wie auch Dritte gegen schädliche Auswirkungen schützen

Bundesamt für Landwirtschaft

Mattenhofstr. 5, 3003 Bern 


\section{Die Aufgaben des BLW im einzelnen}

Dem Amt obliegt auf weiten Gebieten die Vorbereitung der Landwirtschaftsgesetzgebung in einem sehr großen Umfang. Das heißt konkret prüfen, ob neue Vorschriften zur Durchführung der Agrarpolitik nötig sind, stets unter Berücksichtigung rechtsstaatlicher Grundsätze, wie des Subsidiaritäts- und des Verhältnismäßigkeitsprinzips. Sodann sind, meist dem Department, entsprechende Anträge für Bundesgesetze, Bundesbeschlüsse und Verordnungen zu stellen.

Vollzug der Landwirtschaftsgesetzgebung heißt dann demgegenüber etwa:

- Beiträge zusichern und auszahlen - in der Rechnung des BLW sind rund 1,4 Mia Franken an Bundesbeiträgen enthalten - für Forschung, Schulen, Verbesserung der Betriebe, Tierzucht, Viehabsatz, Preisstützung und Absatz von Produkten, Lenkung der Produktion,

- Dienste leisten, im weitesten Sinne, wie etwa im Bereich der Forschung für die Landwirtschaft, der Beschaffung von Grundlagen für die Tierzucht, usw.,

- gebieten und verbieten, wie etwa Bewilligung der Einfuhr von Futtermitteln, Schlachtvieh und Fleisch, von Stallbauten, der Erweiterung der Rebzone, Lenkung der Tierbestände.

Diese Tätigkeiten wären nicht möglich ohne laufende Prüfung der Entwicklung der Landwirtschaft. Die Förderung der Landwirtschaft (v. a. durch Verbesserung der Grundlagen, wie Bildung, Beratung, Verbesserung der Struktur) und ihre Eingliederung in die Gesamtwirtschaft ist dem BLW ausdrücklich übertragen.

\section{Die Organisation des BLW}

Das BLW hatte am 1. Januar 1984 einen Personalbestand mit 1032 besetzten Stellen, die sich wie folgt verteilen:

a) BLW in Bern

b) 7 Forschungsanstalten: Changins (bei Nyon), Liebefeld-Bern, Grangeneuve (bei Freiburg), Reckenholz-Zürich, Wädenswil, Tänikon (bei Aadorf)

c) Eidgenössisches Gestüt in Avenches im gesamten
Die Mitarbeiter des BLW sind auf Kaderstufe vorwiegend Ingenieur-Agronomen und Naturwissenschafter; sodann folgen Volkswirtschafter und einige Juristen.

Dem BLW steht ein Direktor (Ingenieur-Agronom) vor; er ist seinerseits direkt dem Chef des Eidgenössischen Volkswirtschaftsdepartementes unterstellt. Dem Direktor wiederum sind nach der derzeitigen Organisation direkt unterstellt:

- 6 Abteilungen

- eine Stabsstelle Forschungswesen

- die Sektion Berufsbildung und Beratung

- die 7 Forschungsanstalten.

$\mathrm{Zu}$ den einzelnen Abteilungen ist zu sagen:

Die Abteilung Landwirtschaftspolitik befaßt sich überwiegend mit allgemeinen Fragen der Agrarpolitik, mit der Außenwirtschaft und der Entwicklungshilfe. Die Abteilung Pflanzenbau bearbeitet im wesentlichen die planzenbaulichen Fragen, die Futtermittelpolitik und die Investitionskredite. Der Abteilung Viehwirtschaft sind allgemein Zucht, wirtschaftliche Sicherung der viehwirtschaftlichen Produktion und ihres Absatzes (ohne Milch) sowie der Pferdezuchtdienst, das Eidgenössische Gestüt in Avenches, übertragen. Die Abteilung Milch bearbeitet Fragen der Produktion und der Verwertung der Milch und der Milchprodukte, mit Einschluß der Preissicherung und der Lenkung, wie etwa mit der Milchkontingentierung. Bodenverbesserungen, wie Güterzusammenlegungen, und landwirtschaftliche Hochbauten fallen in das Aufgabengebiet des Eidgenössischen Meliorationsamtes. Die Abteilung Verwaltungsdienste befaßt sich im wesentlichen mit dem Finanzdienst, wie Budget, Staatsrechnung, mit dem Personaldienst, mit Organisationsfragen; zu dieser Abteilung gehören der Informationsdienst und ein allgemeiner Rechtsdienst. Die Forschungsanstalten erarbeiten wissenschaftliche und technische Grundlagen der Produktion in der Landwirtschaft und unterstützen sie in der volkswirtschaftlichen Aufgabe, v. a. durch Förderung der Produktivität und der Qualität, unter angemessener Berücksichtigung der ökologischen Gesichtspunkte.

Eng verbunden mit dem BLW ist das Eidgenössische Ernährungsamt, und zwar aufgabenmäßig, personell und organisatorisch. Dieses Amt wird dann aktiv, wenn die Versorgung des Landes mit lebenswichtigen Gütern erheblich bedroht wird, beispielsweise durch Boykott oder andere internationale Krisenlagen.

Dr. A. Pfenninger 